\title{
Efficacy of Dexamethasone for Acute Primary Immune Thrombocytopenia Compared to Prednisolone: A Systematic Review and Meta-analysis
}

\author{
Yasuyuki Arai ${ }^{1,2}$ Hiroyuki Matsui ${ }^{1}$ Tomoyasu Jo ${ }^{1}$ \\ ${ }^{1}$ Department of Hematology and Oncology, Graduate School of \\ Medicine, Kyoto University, Kyoto, Japan \\ ${ }^{2}$ Laboratory of Host Defenses, National Institute of Allergy and \\ Infectious Diseases, National Institutes of Health, Bethesda, \\ Maryland, United States
}

\author{
Tadakazu Kondo $^{1} \quad$ Akifumi Takaori-Kondo ${ }^{1}$
}

TH Open 2017;1:e73-e81.

\begin{abstract}
Address for correspondence Tadakazu Kondo, MD, PhD, Department of Hematology and Oncology, Graduate School of Medicine, Kyoto University, 54, Shogoin Kawahara-cho, Sakyo-ku, Kyoto, 606-8507, Japan (e-mail: tadakazu@kuhp.kyoto-u.ac.jp).
\end{abstract}

\begin{abstract}
Keywords

- thrombocytopenia

- dexamethasone

- prednisolone

- meta-analysis

Corticosteroids have been established as first-line therapy in acute primary immune thrombocytopenia (ITP), and the clinical guidelines recommend either dexamethasone (Dex) or prednisolone (PSL). The types and dosages of corticosteroids, however, have not yet been determined, because previous randomized control trials (RCTs) comparing Dex and PSL showed controversial results in terms of efficacy. To understand and interpret all available evidence, we conducted a systematic review and meta-analysis of RCTs. The main outcome measure was the incidence of sustained response (SR; platelet count $>30 \times 10^{9} / \mathrm{L}$ for 6 months without concomitant treatments after the completion of the final therapies). Eight RCTs (totaling 704 patients) were included in this study. The incidence of SR showed no significant difference, while it was significantly higher in the Dex arm when used with posttherapy (more than one course of Dex or tapering corticosteroids added; risk ratio [RR], $1.82 ; 95 \%$ confidence interval $[\mathrm{Cl}], 1.38-2.41 ; p<0.01)$. A single course of Dex showed no significant difference. The overall response (platelet $>30 \times 10^{9} / \mathrm{L}$ ) at day 28 was significantly improved in the Dex arm (RR, 1.11; $95 \% \mathrm{Cl}, 1.01-1.22 ; p=0.03)$ and Dex with posttherapy suppressed long-term relapse (RR of nonevent, 1.32; $95 \% \mathrm{Cl}, 1.10-1.59$; $p<0.01)$. There were significantly fewer adverse events in the Dex arm (RR, $0.45 ; 95 \% \mathrm{Cl}$, $0.37-0.55 ; p<0.01)$. Use of Dex with posttherapy instead of PSL may be more beneficial as the initial therapy. Studies comparing Dex with other new strategies are essential to determine the most suitable therapeutic regimens for acute ITP.
\end{abstract}

\section{Introduction}

Acute primary immune thrombocytopenia (ITP) is characterized by acquired immune-mediated peripheral platelet destruction and impaired platelet production in the bone marrow $^{1}$ with consequent increased risk of bleeding. ${ }^{2}$ The pathogenesis of ITP is not completely understood, but the production of autoreactive antibodies against platelet antigens may be involved in platelet destruction and impaired platelet production. $^{3}$
Corticosteroids are the established first-line therapy in symptomatic ITP. Previous guidelines recommended either high-dose dexamethasone (Dex) or prednisolone (PSL). ${ }^{4,5}$ The types and dosages of corticosteroids, however, have not yet been determined, because previous randomized control trials (RCTs) comparing Dex and PSL showed controversial results in terms of the efficacy. Cui et $\mathrm{al}^{6}$ and Mashhadi et $\mathrm{al}^{7}$ showed the superiority of Dex in terms of long-term sustained response (SR), while Li et $\mathrm{al}^{8}$ and Bae et $\mathrm{al}^{9}$ showed inferior SR in the Dex arm. Moreover, the 
newest and the largest study from Wei et $\mathrm{al}^{10}$ indicated that Dex and PSL were equivalent in terms of SR.

A recent systematic review and meta-analysis on this topic (Dex vs. PSL) summarized these five controversial RCTs $(N=553)$, concluding that Dex did not improve SR compared with PSL. ${ }^{11}$ This analysis, however, had several limitations, such as the inclusion criteria (only literature written in English was included) and lack of subgroup analysis of the corticosteroid dosage (due to the small number of included studies). Among the excluded studies, there were at least two large-sized RCTs published in Chinese (total $N=153)^{6,8}$ If included, they might have had enough power to amend the final conclusions of the meta-analysis and enable the various subgroup analyses.

Therefore, to understand and interpret all available evidence including the previously excluded RCTs, we conducted a systematic review and meta-analysis, and evaluated the efficacy (long-term SR, short-term overall response [OR], and relapse) and safety (short- and long-term adverse events) of Dex compared with PSL as an initial therapy for ITP.

\section{Methods}

\section{Data Sources and Searches}

We conducted a literature search to identify all published and unpublished RCTs based on the search strategies suggested in the Cochrane Handbook for Systematic Reviews of Interventions. We performed a search of the following electronic databases: MEDLINE (via PubMed) (1950 to January 2017) and Cochrane Central Register of Controlled Trials (CENTRAL; The Cochrane Library 2016, Issue 12). The search strategies are outlined in -Tables $\mathbf{S 1}$ and $\mathbf{S 2}$, respectively (supplementary tables available in the online version only). We also searched unpublished clinical trials, using ClinicalTrials.gov, and conference proceedings of the American Society of Hematology (ASH; 2004-2016).

The reference lists of all the included studies and relevant systematic reviews were assessed to identify additional studies missed in the original electronic searches. A citation search was also conducted through Web of Science to identify articles citing any of the included studies.

\section{Study Selection}

We included all relevant RCTs in all languages. We also included abstracts and unpublished data, if sufficient information on the study design, participant characteristics, interventions, and outcomes were available. Patients were limited to those with newly diagnosed acute primary ITP; those with secondary thrombocytopenia or with previous therapeutic interventions for ITP were basically excluded. There were no limitations for age or sex. Participants could be outpatients or hospital inpatients at the time of enrollment.

Only RCTs that compared an experimental group receiving Dex (any dose with any frequency and any therapeutic duration) to a control group receiving PSL (any dose with any frequency and any therapeutic duration) were included. RCTs using prednisone, instead of PSL, were also included.
Two review authors (Y.H. and H.M.) scanned the titles and abstracts of the studies identified by the electronic search strategies to assess their eligibility. The two authors then independently evaluated the full-text versions for each potentially relevant study for inclusion in the meta-analysis. Disagreements between authors were resolved by discussion. If necessary, arbitration was provided by the senior authors (T.K. and A.T-K.). When missing information inhibited the evaluation of a study, further information was sought from the original authors or other possible sources. The study selection process is reported in a PRISMA flow diagram (-Fig. S1, supplementary figure available in the online version only).

\section{Data Extraction and Quality Assessment}

Data from the included trials were independently extracted by two review authors (Y.A. and T.J.) from the included trials using a structured, pilot-tested, data extraction form (- Table S3, supplementary table available in the online version only). Differences in data extraction were resolved either by discussion or by consultation with the senior authors (T.K. and A.T-K.).

These two review authors also independently assessed the eligible studies for bias using the tool described in the Cochrane Handbook for Systematic Reviews of Interventions. We evaluated the risk of bias as low, high, or unclear using an assessment form designed for the topic of this review (-Table 54, supplementary table available in the online version only). Any disagreements were discussed with the senior authors until a consensus was obtained.

\section{Data Synthesis and Analysis}

The primary outcome was the incidence of SR (platelet count [Plt] $>30 \times 10^{9} / \mathrm{L}$ for 6 months without concomitant treatments after the completion of the final therapy); the secondary outcomes included the OR (Plt $>30 \times 10^{9} / \mathrm{L}$ ) and complete response (CR; Plt $\left.>100 \times 10^{9} / \mathrm{L}\right)$ at an early time point (on day 14 or 28 of the first therapy), relapse after therapy (loss of response or bleeding episodes), and the incidence of adverse effects.

All of our treatment effects were measured as dichotomous data, and were presented as the summaries of the risk ratios (RRs) with 95\% confidence intervals (CIs).

If the number of included RCTs was sufficient enough, we planned to explore potential publication bias by generating a funnel plot and performing a linear regression test (considering a $p$-value $<0.1$ as significant).

If these data were sufficiently similar, we performed a meta-analysis using RevMan software (version 5.3; Copenhagen: the Nordic Cochrane Centre, the Cochrane Collaboration, 2014). We used a fixed-effect model (the Mantel-Haenszel method) for pooled analysis of data because relatively similar results were expected in our systematic review. These results were also confirmed in the random-effects model.

Finally, we explored the potential sources of heterogeneity using the subgroup of the Dex regimen: "Dex monotherapy" (only one course of Dex without any additional corticosteroid) versus "Dex with posttherapy" (two or more courses of Dex, or any consolidation or tapering corticosteroid therapy after Dex administration, irrespective of the initial response). If the 
outcome in the subgroup was not determined from a study, we included it in the subgroup for which more than $80 \%$ of participants fulfilled the criteria.

\section{Results}

\section{Identification of Studies}

The study identification and selection process are illustrated in -Fig. S1 (supplementary figure available in the online version only). The primary search yielded 157 citations, of which 7 RCTs met the inclusion criteria. ${ }^{6-8,10,12-14}$ A search of conference proceedings revealed one additional relevant study (abstract only). ${ }^{9}$ In total, we included eight RCTs totaling 704 patients with acute primary ITP $(\kappa=0.83)$. All the RCTs included only adult patients; children younger than 16 years were not included. One study included relapsed ITP cases ( 5 out of 36 cases), ${ }^{12}$ and in the other study ${ }^{14} 3$ patients (out of total 22 patients) were secondary ITP due to lymphoid malignancy. Secondary thrombocytopenia patients due to hepatitis viral infection, systemic lupus erythematosus, and pregnancy were clearly excluded in these RCTs. In one RCT from Matschke et al, ${ }^{14}$ all patients were administrated prednisone $(1 \mathrm{mg} / \mathrm{kg} /$ day $)$ for 7 days before being randomized; we included this report in our analysis because we considered the differences in the outcomes between the groups to be derived from the effects of Dex versus PSL after randomization. Individual patient data were not available in any studies.

\section{Study Characteristics}

The studies were conducted mainly in Asian countries (China, Korea, and Thailand) as well as in Iran and Germany, and were published between 2009 and 2016 ( - Table 1). The median age of the participants ranged from 24 to 46 years. The intervention groups (Dex) consisted of high-dose Dex ( $40 \mathrm{mg} /$ day for 4 days in most studies; $0.6 \mathrm{mg} / \mathrm{kg} /$ day for 4 days in one study ${ }^{14}$ ). In three studies, patients were administered Dex monotherapy (one course of Dex; additional courses were added only in nonresponders) without any consolidation or tapering of the corticosteroid administration, ${ }^{8-10}$ while in five studies, patients were administered Dex with posttherapy (two to six courses of Dex for all patients ${ }^{6,13,14}$ or low-dose Dex ${ }^{13}$ or $\mathrm{PSL}^{7,12}$ accompanying the preceding courses of Dex, irrespective of the treatment effects in the first course of Dex). Total dosage of corticosteroids in the interventional arm were calculated (estimating the body weight was $40-80 \mathrm{~kg}$ ), and converted to the equivalent amounts of PSL ( - Table 1 and $\mathbf{- T a b l e} \mathbf{5 5}$ [supplementary table available in the online version only]). All comparison groups were administered PSL (1-1.5 $\mathrm{mg} / \mathrm{kg} / \mathrm{day}$ ) for 14 to 28 days followed by gradually tapered PSL dose. Prednisone, instead of PSL, was used in four RCTs, ${ }^{6,10,13,14}$ both of which were analyzed together as the PSL arm. Crossover from one arm to the other was allowed in two studies ${ }^{12,14}$ (-Table $\mathbf{1}$ ), where we used intention-to-treat analysis. Intravenous immunoglobulin, ${ }^{12}$ rituximab, ${ }^{13}$ and/or thrombopoietin receptor agonist ${ }^{13}$ were used in some studies for poor responders of these corticosteroid studies.

\section{Risk of Bias in Included Studies}

The risk of bias is graphically summarized in - Fig. 52 $(\kappa=0.72$; supplementary figure available in the online version only). All the studies lacked sufficient blinding of participants and personnel (classified as "high risk"), which can work as a potential bias to the intervention group (Dex arm).

\section{Publication Bias}

Publication bias was assessed using a funnel plot for the OR. The results showed a symmetric distribution, indicating a low likelihood of publication bias ( $p=0.71$; - Fig. S3, supplementary figure available in the online version only).

\section{Outcomes}

\section{Sustained Response}

We analyzed the SR (Plt $\left.>30 \times 10^{9} / \mathrm{L}\right)$ at 6 months after the completion of therapies as a dichotomous outcome, because time-to-event data were available only in one RCT. ${ }^{14}$ Seven RCTs with 668 patients presented data on SR. One study ${ }^{14}$ defined SR as Plt $>50 \times 10^{9} / \mathrm{L}$, and the RR shown in this study was integrated in our analysis as it was. The pooled results demonstrated no significant difference between the two arms (RR, 1.13; 95\% CI, 0.94-1.36; $p=0.21$; - Fig. 1a ) ${ }^{6-10,13,14}$ This tendency was the same if analyzed with the random-effects model (RR, 1.23; 95\% CI, 0.84-1.79; $p=0.28$ ).

Subgroup analyses showed that four studies using Dex with posttherapy (two or more courses of Dex in all patients irrespective of the initial response, or Dex administration followed by consolidation and/or tapered corticosteroid therapy $)^{6,7,13,14}$ showed a significantly higher incidence of SR compared with that of the PSL arm $(N=231 ; \mathrm{RR}, 1.82$; 95\% CI, 1.38-2.41; $p<0.01 ;-$ Fig. 1b). If the random-effects model was used, RR was 1.78 (95\% CI, 1.37-2.32; $p<0.01$ ). On the other hand, three studies using Dex monotherapy (only one course of Dex; additional courses of Dex were administered only to nonresponders $)^{8-10}$ showed inferior SR incidence compared with that of PSL with borderline significance $(N=437 ; \quad R R, \quad 0.80 ; \quad 95 \% \quad \mathrm{Cl}, \quad 0.62-1.03$; $p=0.08$; - Fig. 1b). RR was 0.79 (95\% Cl, 0.59-1.08; $p=0.14$ ) if the random-effects model was introduced. All the RCTs included in the Dex monotherapy subgroup used a smaller amount of corticosteroid $(1,000 \mathrm{mg}$ or less if converted to PSL), while studies in the Dex with posttherapy subgroup involved a larger amount of steroid (more than $\mathbf{1 , 0 0 0} \mathrm{mg}$ if converted to PSL; - Table $\mathbf{1}$ and - Table S5 [supplementary table available in the online version only]).

Data on SR at 1 year was obtained from five RCTs, $7,8,10,13,14$ and the Dex arm showed the significantly superior SR compared with PSL $(N=458$; RR, $1.54 ; 95 \% \mathrm{Cl}, 1.17-2.03$; $p=0.01$ ) if analyzed in the fixed-effect model, and the difference was with borderline significance if analyzed with the random-effects model (RR, 1.61; 95\% CI, 0.93-2.79; $p=0.09$ ).

\section{Early Overall Response and Complete Response}

We compared the early OR (Plt $>30 \times 10^{9} / \mathrm{L}$ ) data within 28 days after the initial therapies. As shown in - Fig. 2a, data regarding the incidence of early OR were extracted 


\begin{tabular}{|c|c|c|c|c|c|c|c|c|}
\hline 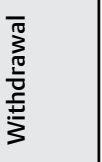 & 0 & $\frac{\underline{v}}{m}$ & 0 & 0 & $\underset{\forall}{\equiv}$ & 0 & $\underset{-}{\underline{Q}}$ & $\underset{\sim}{\underline{v}}$ \\
\hline 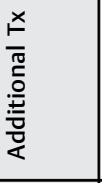 & \begin{tabular}{|l}
$\hat{\equiv}$ \\
$\vdots$ \\
$\underline{u}$ \\
$\geq$
\end{tabular} & 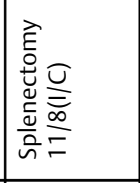 & 0 & 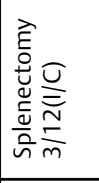 & 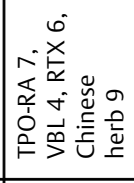 & 0 & 0 & 0 \\
\hline 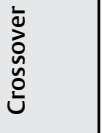 & $\begin{array}{l}\overline{0} \\
\dot{0} \\
\frac{u}{m}\end{array}$ & 0 & 0 & 0 & 0 & 0 & 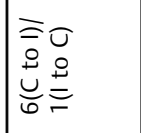 & 0 \\
\hline 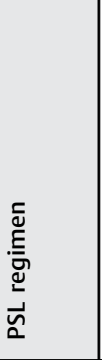 & 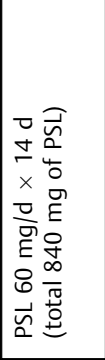 & 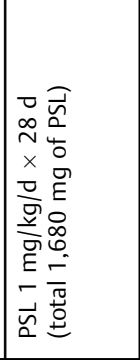 & 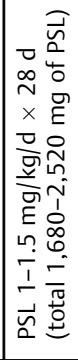 & 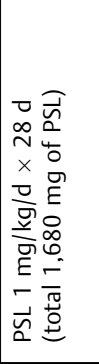 & 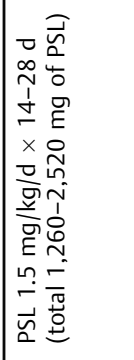 & 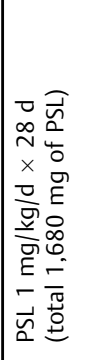 & 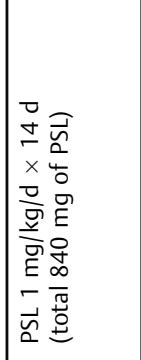 & 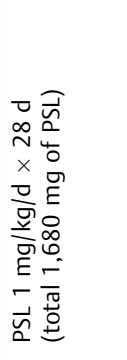 \\
\hline 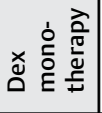 & $z$ & $>$ & z & z & $>$ & $z$ & $z$ & $>$ \\
\hline 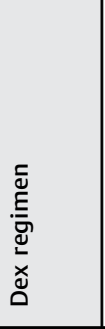 & 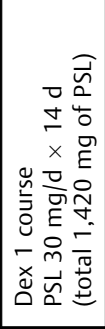 & 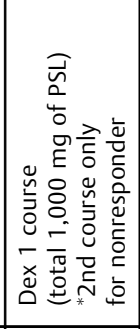 & 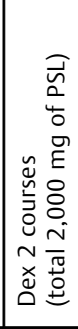 & 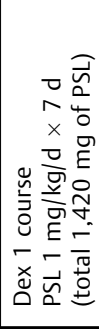 & 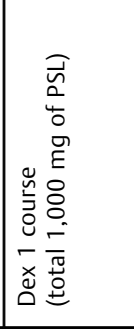 & 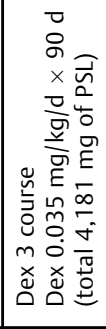 & 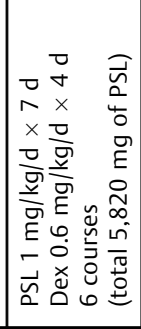 & 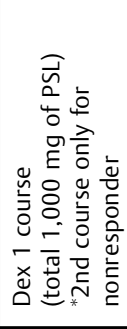 \\
\hline 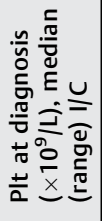 & 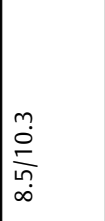 & $\frac{F}{6}$ & 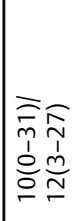 & 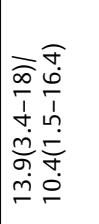 & 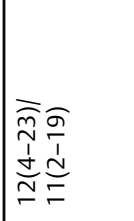 & 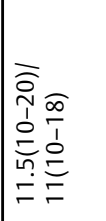 & 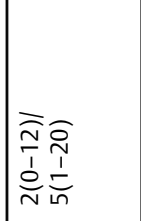 & 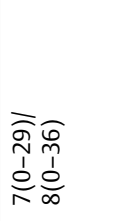 \\
\hline 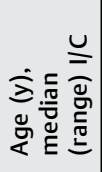 & 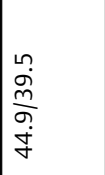 & $\frac{f}{8}$ & 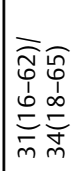 & 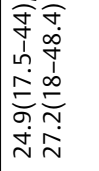 & 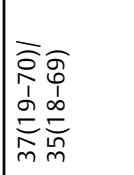 & 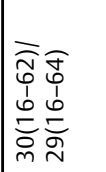 & 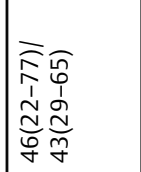 & 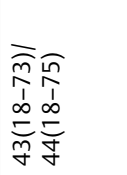 \\
\hline ঐ & $\frac{\infty}{\infty}$ & $\mid \frac{8}{\stackrel{8}{9}}$ & $\frac{\hat{m}}{\stackrel{a}{\sim}}$ & $\frac{\hat{y}}{\underline{m}}$ & $\frac{\mathscr{h}}{\frac{h}{\infty}}$ & $\frac{\rho}{\frac{h}{q}}$ & $\frac{a}{m}$ & $\begin{array}{l}\stackrel{0}{0} \\
\frac{m}{i} \\
i n\end{array}$ \\
\hline है & 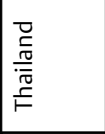 & 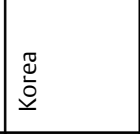 & 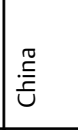 & 胥 & 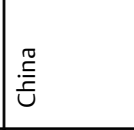 & 冚 & 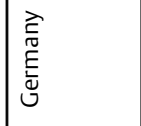 & 苞 \\
\hline 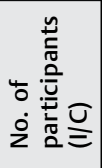 & $\mid \begin{array}{l}\infty \\
\stackrel{\infty}{\infty} \\
\infty \\
\\
0 \\
n\end{array}$ & 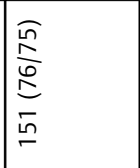 & 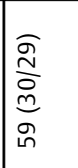 & 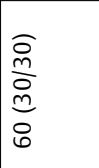 & $\begin{array}{l}\frac{\partial}{g} \\
\frac{v g}{g} \\
\sigma \\
\sigma\end{array}$ & $\begin{array}{l}\frac{\widehat{2}}{\frac{N}{\sigma}} \\
\frac{6}{6}\end{array}$ & $\begin{array}{l}\frac{a}{a} \\
\underline{n} \\
N\end{array}$ & 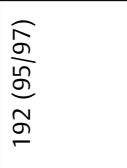 \\
\hline 号 & 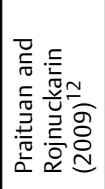 & 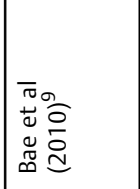 & 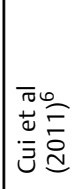 & 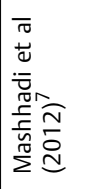 & 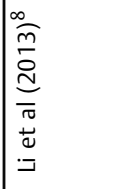 & 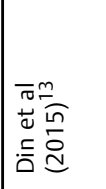 & 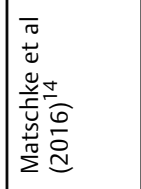 & 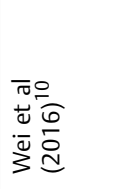 \\
\hline
\end{tabular}


a sustained response

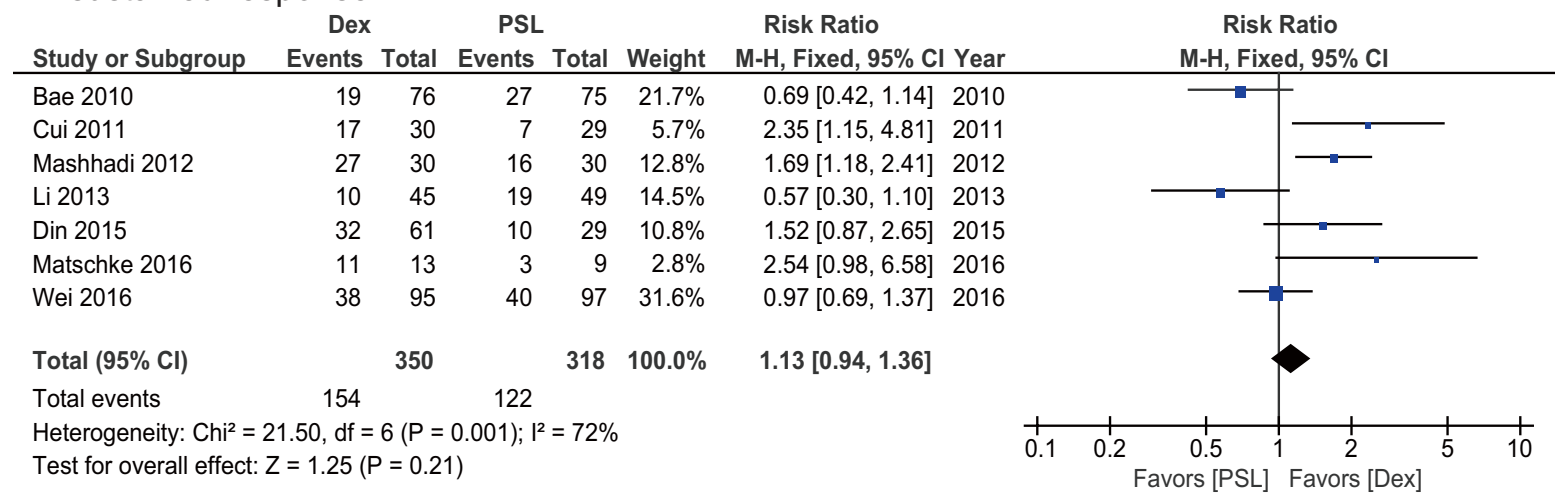

b sustained response (subgroup analysis)

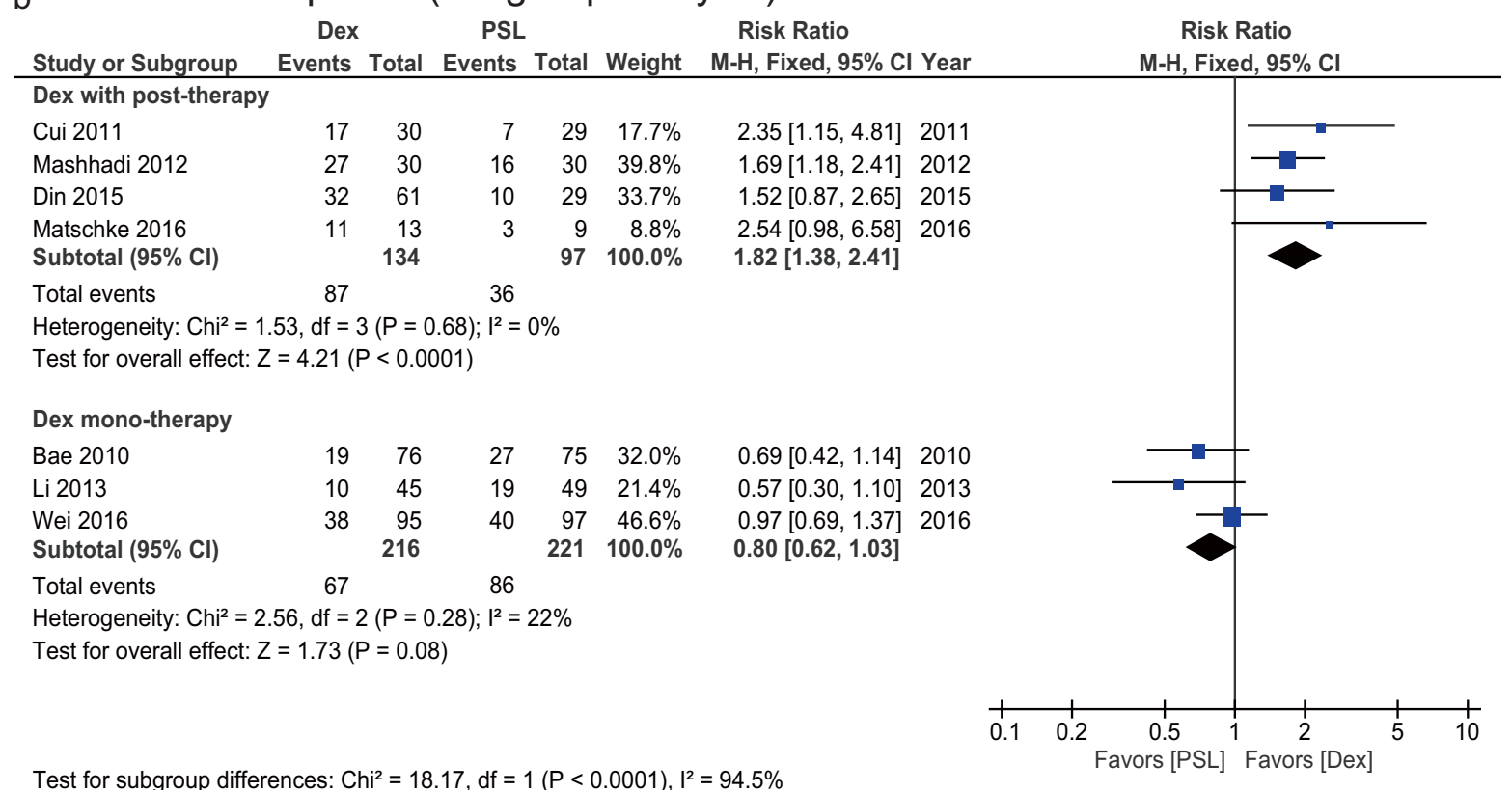

Fig. 1 Forest plots of long-term sustained response (SR) comparison. The summary effect estimate (risk ratio [RR] of SR at 6 months; platelet counts $>30 \times 10^{9} / \mathrm{L}$ ) for individual randomized controlled trials (RCTs; dexamethasone [Dex] vs. prednisolone [PSL]) are indicated with black boxes (their sizes are proportional to the study weight), with the lines indicating 95\% confidence intervals (Cls). The overall summary effect estimate (RR) and 95\% $\mathrm{Cl}$ are indicated by the diamond below. (a) SR in all the RCTs and (b) subgroup analyses (Dex with posttherapy and Dex monotherapy).

from all eight studies, including a total of 704 patients. The pooled results indicated that Dex significantly increased early $\mathrm{OR}(\mathrm{RR}, 1.11 ; 95 \% \mathrm{CI}, 1.01-1.22 ; p=0.03)$. The random-effects model indicated the same tendency $(\mathrm{RR}, 1.19 ; 95 \% \mathrm{CI} 1.04-$ 1.37; $p=0.01$ ). We also calculated the incidence of $\mathrm{OR}$ including only RCTs which showed OR at an earlier time point (14 days after therapy). In this analysis, the difference in OR was more prominent (six RCTs; $N=459$; RR, 1.29; $95 \% \mathrm{CI}$, 1.13-1.46; $p<0.01$;-Fig. 2b), while RR was 1.26 (95\% CI, $1.13-1.41 ; p<0.01)$ if analyzed with the random-effects model.

Data on early CR (Plt $\left.>100 \times 10^{9} / \mathrm{L}\right)$ at 28 days were extracted from two RCTs $(N=252) ;{ }^{7,10}$ the pooled results showed significantly superior results in the Dex arm both with the fixed-effect model (RR, 2.06; 95\% CI, 1.52-2.78; $p<0.01$; - Fig. 2c) and with the random-effects model (RR, 2.09; 95\% CI, 1.54-2.82; $p<0.01$ ).

\section{Relapse}

We evaluated the incidence of relapse as a dichotomous outcome. Time-to-event data were not available except for one study. ${ }^{14}$ In each study, relapse was defined as the loss of response or the appearance of bleeding episodes, and the judgement of relapse was not related to whether the secondary therapeutic intervention was initiated or not. As shown in - Fig. 2d, data regarding relapse were extracted from all eight studies (including 542 patients who had once achieved OR). The pooled results showed that the incidence was not significantly different between the two arms (RR of nonevent, $1.07 ; 95 \% \mathrm{CI}, 0.94-1.22$; $p=0.29$ ), while RR was 1.08 (95\% CI, $0.91-1.28 ; p=0.36$ ) in the random-effects model. Subgroup analyses showed that Dex with posttherapy significantly reduced relapse compared with PSL arm (five RCTs; $N=219$; RR of nonevent, $1.32 ; 95 \%$ CI, 1.10-1.59; $p<0.01$ with the fixed-effect model 
e78 Meta-analysis of Dexamethasone vs. Prednisolone for Acute ITP Arai et al.

a overall response at 28 days

\begin{tabular}{|c|c|c|c|c|c|c|c|c|c|}
\hline Study or Subgroup & \multicolumn{2}{|l|}{ Dex } & \multicolumn{2}{|l|}{ PSL } & \multicolumn{3}{|c|}{ Risk Ratio } & \multicolumn{2}{|c|}{$\begin{array}{c}\text { Risk Ratio } \\
\text { M-H, Fixed, } 95 \% \mathrm{CI}\end{array}$} \\
\hline Praituan 2009 & 17 & 18 & 11 & 18 & $4.9 \%$ & $1.55[1.05,2.27]$ & 2009 & & \\
\hline Bae 2010 & 52 & 76 & 62 & 75 & $27.8 \%$ & $0.83[0.69,1.00]$ & 2010 & 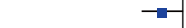 & \\
\hline Cui 2011 & 15 & 30 & 7 & 29 & $3.2 \%$ & $2.07[0.99,4.33]$ & 2011 & & \\
\hline Mashhadi 2012 & 30 & 30 & 24 & 30 & $10.9 \%$ & $1.24[1.03,1.50]$ & 2012 & & $\square$ \\
\hline Li 2013 & 30 & 45 & 34 & 49 & $14.5 \%$ & $0.96[0.73,1.27]$ & 2013 & & - \\
\hline Din 2015 & 25 & 61 & 8 & 29 & $4.8 \%$ & $1.49[0.77,2.88]$ & 2015 & & \\
\hline Matschke 2016 & 11 & 13 & 8 & 9 & $4.2 \%$ & $0.95[0.69,1.32]$ & 2016 & & - \\
\hline Wei 2016 & 78 & 95 & 67 & 97 & $29.6 \%$ & $1.19[1.01,1.40]$ & 2016 & & - \\
\hline Total $(95 \% \mathrm{Cl})$ & & 368 & & 336 & $100.0 \%$ & $1.11[1.01,1.22]$ & & & 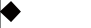 \\
\hline Total events & 258 & & 221 & & & & & & \\
\hline $\begin{array}{l}\text { Heterogeneity: } \mathrm{Ch}^{2}= \\
\text { Test for overall effect }\end{array}$ & $\begin{array}{l}20.07, d f= \\
Z=2.13(\end{array}$ & $\begin{array}{l}=7(P= \\
P=0.03\end{array}$ & $\begin{array}{l}0.005) ; 1^{2} \\
\text { 3) }\end{array}$ & $65 \%$ & & & & \begin{tabular}{c|}
1 \\
0.5 \\
Favors [PSL]
\end{tabular} & $\begin{array}{r}1 \\
2 \\
\text { Favors [De }\end{array}$ \\
\hline
\end{tabular}

b overall response at 14 days

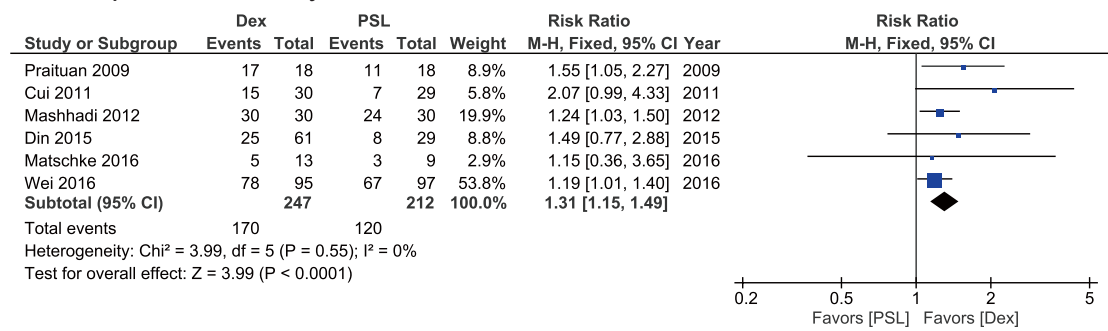

C complete response at 28 days

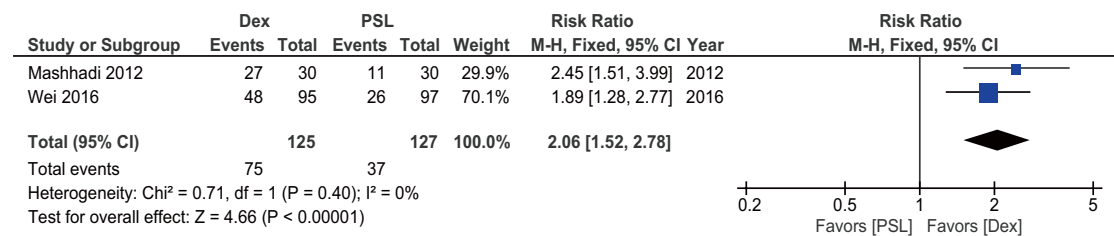

d relapse

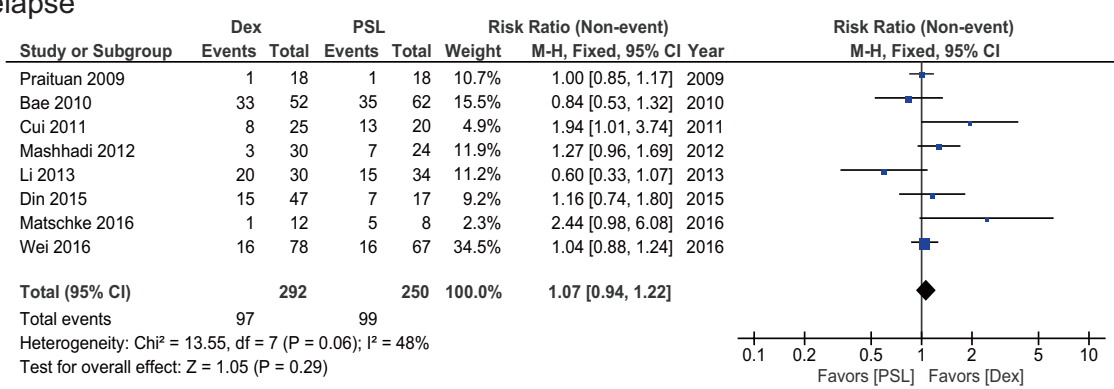

e relapse (subgroup analysis)

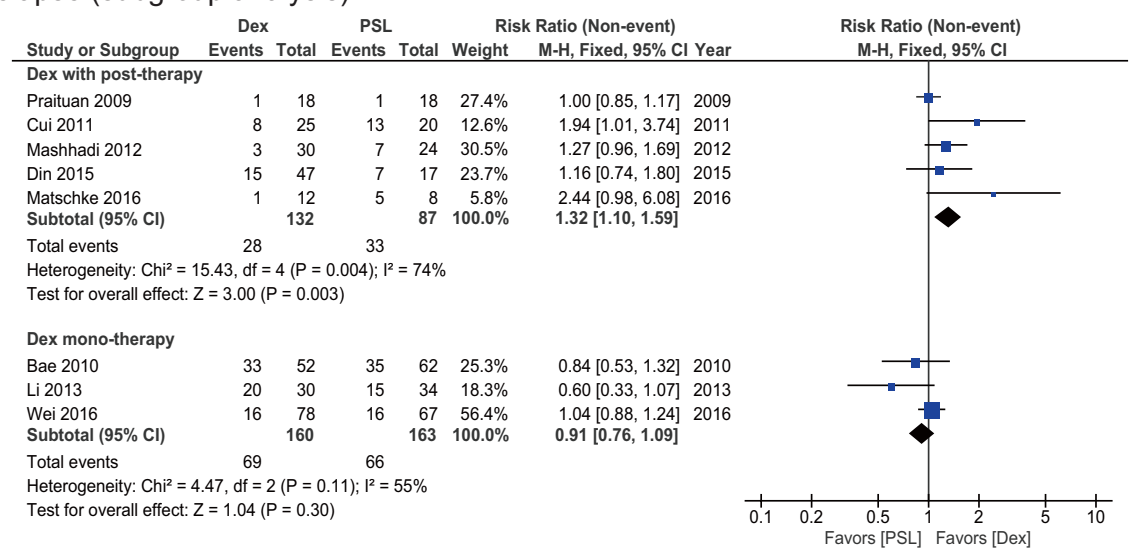

Fig. 2 Forest plots of short-term overall response (OR), complete response (CR), and relapse comparison. The summary effect estimate (risk ratio [RR] of overall response [OR; platelet counts $>30 \times 10^{9} / \mathrm{L}$ ] and complete response [CR; platelet counts $\left.>100 \times 10^{9} / \mathrm{L}\right]$ at 28 or 14 days after the initial therapy) for individual randomized controlled trials (RCTs; dexamethasone [Dex] vs. prednisolone [PSL]) are indicated with black boxes (their sizes are proportional to the study weight), with the lines indicating $95 \%$ confidence intervals (Cls). The overall summary effect estimate (RR) and $95 \% \mathrm{Cl}$ are indicated by the diamond below for OR at 28 days (a) and 14 days (b), and CR at 28 days (c). Risk ratio of nonevent regarding posttherapeutic relapse (loss of response or bleeding episodes) was indicated in all RCTs (d) and in each subgroup regarding the addition of posttherapy in the Dex arm (e). 
[ - Fig. 2e], and RR, 1.39; 95\% CI, 1.06-1.82; $p=0.02$ with the random-effects model), while Dex monotherapy did not induce any significant reduction (three RCTs; $N=323$; RR of nonevent, $0.91 ; 95 \% \mathrm{CI}, 0.76-1.09 ; p=0.30$ with the fixedeffect model [ - Fig. 2e], and RR, 0.87; 95\% CI, 0.63-1.22; $p=0.43$ with the random-effects model).

\section{Incidence of Adverse Events}

All but one $e^{8}$ of the studies supplied information about acute- or chronic-phase adverse events related to corticosteroid administration ( $N=323$ in the Dex arm; $N=287$ in the PSL arm). However, information on the grade of each adverse event was not sufficient for analysis. In the Dex arm, psychiatric symptoms (including anxiety, insomnia, and restlessness) were the most prominent $(N=26 ; 8.0 \%)$, followed by hyperglycemia $(N=18 ; 5.6 \% ;-$ Table 2). In comparison, Cushing's syndrome was most often observed in the PSL arm $(N=43 ; 15.0 \%)$, followed by gastrointestinal symptoms including nausea and dyspepsia $(N=25 ; 8.7 \%$; - Table 2$)$. In total, the Dex arm showed significantly lower incidence of all adverse events $(N=90 ; 27.9 \%)$ compared with that of the PSL arm $(N=177$; 61.7\%) (RR, 0.45; 95\% CI, 0.37-0.55; $p<0.01$ ). Number of patients on whom therapeutic interventions were withdrawn is shown in -Table 1.

This lower incidence of adverse events in the Dex arm was confirmed in both subgroups of the Dex regimen. The Dex with posttherapy subgroup showed a relatively higher incidence (32.9\%) compared with that of the Dex monotherapy group (23.4\%), but both subgroups showed significantly lower incidence when compared with that of the PSL arm (RR, 0.39; 95\% CI, 0.31-0.50; $p<0.01$, and RR, 0.50; 95\% CI, $0.36-0.68 ; p<0.01$, respectively). Only one $\mathrm{RCT}^{10}$ provided data on adverse events in the subgroup of elderly, and significant increase in elderly was not observed.

Table 2 Summary of adverse events

\begin{tabular}{|l|l|l|l|l|}
\hline \multirow{2}{*}{ Events } & \multicolumn{2}{|l|}{$\begin{array}{l}\text { Dex arm } \\
(N=323)\end{array}$} & \multicolumn{2}{l|}{$\begin{array}{l}\text { PSL arm } \\
(N=287)\end{array}$} \\
\cline { 2 - 5 } & $N$ & $\%$ & $N$ & $\%$ \\
\hline Transaminase increase & 6 & 1.9 & 6 & 2.1 \\
\hline Hyperglycemia & 18 & 5.6 & 18 & 6.3 \\
\hline Hypokalemia & 3 & 0.9 & 1 & 0.3 \\
\hline Acne & 4 & 1.2 & 6 & 2.1 \\
\hline Cushing's syndrome & 0 & 0.0 & 43 & 15.0 \\
\hline Weight gain & 5 & 1.5 & 23 & 8.0 \\
\hline Hypertension & 10 & 3.1 & 14 & 4.9 \\
\hline Gastrointestinal symptoms & 9 & 2.8 & 25 & 8.7 \\
\hline Psychiatric symptoms & 26 & 8.0 & 15 & 5.2 \\
\hline Infection & 3 & 0.9 & 7 & 2.4 \\
\hline Edema & 3 & 0.9 & 7 & 2.4 \\
\hline Arthralgia/myalgia & 0 & 0.0 & 3 & 1.0 \\
\hline Dizziness/fatigue & 3 & 0.9 & 9 & 3.1 \\
\hline Total & 90 & 27.9 & 177 & 61.7 \\
\hline
\end{tabular}

\section{Discussion}

This systematic review and meta-analysis of the efficacy of high-dose Dex for newly diagnosed acute primary ITP included eight trials with 704 randomly assigned participants. Our main results showed no significant differences regarding SR at 6 months, while SR was significantly higher in the Dex arm if used with posttherapy (more than one course of Dex or with an additional tapering dose of corticosteroids). Dex also improved early OR within 14 or 28 days, and Dex with posttherapy decreased long-term relapse compared with PSL. Therapyrelated adverse events were observed with less frequency in the Dex arm.

Our analyses on SR suggested that Dex with posttherapy may have the superiority compared with PSL. These data were not included in the previous meta-analysis ${ }^{11}$ and are newly revealed in the subgroup analyses of the present study. We used the fixed-effect model to synthesize SR data because of the low heterogeneity in each subgroup $\left(I^{2}=0 \%\right.$ in Dex with posttherapy; $I^{2}=22 \%$ in Dex monotherapy); the same conclusions were obtained if we used the random-effects model.

Perturbation in the definition of SR may have existed throughout this meta-analysis. In three studies, ${ }^{7-9}$ definition of SR was not clearly mentioned, and, therefore, it is possible that these studies treated the SR rate at 6 months from the beginning of the initial therapy, instead of that without concomitant treatments (i.e., periods from the end of the last therapy, as defined in this meta-analysis). This uncertainty in the definition can be a bias for SR analysis. Therefore, we performed the sensitivity analysis excluding these three studies (including only five RCTs defining SR clearly as we defined), and still confirmed the superiority of Dex with posttherapy compared with PSL (RR, 1.75; 95\% CI, 1.31-2.35; $p<0.01$ ).

Moreover, two studies included small number of patients with refractory or secondary ITP. ${ }^{12,14}$ We analyzed SR or other outcomes without excluding these studies (because these patients existed in the small proportion of the whole cohort in each study). We confirmed that the sensitivity analysis excluding these studies led to the same conclusion mentioned earlier (RR, 1.75; 95\% CI, 1.31-2.35; $p<0.01$ for SR; RR, 1.10 ; 95\% CI, $0.99-1.21 ; p=0.09$ for OR).

Thus, our findings suggest that Dex with posttherapy may possibly be one of the first-line therapeutic choices, instead of PSL or Dex as a monotherapy. This suggestion depends on the highly reliable results of SR (confirmed by various models and sensitivity analyses), and is also supported by our secondary endpoints, including the significantly higher OR in the Dex arm and lower frequency of relapse in the subgroup of Dex with posttherapy, which may have led to the superior SR observed in the current study.

The initial response in the early time point to corticosteroid is also essential in the treatment of ITP, and Dex was more desirable in this viewpoint. The Dex arm demonstrated significantly higher RR than that of the PSL arm at 28 days; this difference was more prominent at 14 days after the initiation of therapy. These results not only reveal a higher OR in the Dex arm, but the data also suggest that Dex has the potential to restore platelets much faster than PSL. These findings are 
compatible with those of previous studies. Cheng et al observed platelet recovery as early as day 3 of Dex administration in a single arm study, ${ }^{15}$ while Alpdogan et al reported a median response of 8.4 days after PSL treatment. ${ }^{16}$ In vitro analysis also supported rapid platelet recovery with Dex by showing that Dex enhanced the expression of inhibitory Fcr receptors on monocytes within 4 days, and normalized the perturbed balance of stimulatory/inhibitory $\mathrm{Fcr}$ receptors, which has been implicated in the pathogenesis of ITP. ${ }^{17}$

Thus, Dex can restore platelets more rapidly and more frequently in ITP, but our data suggested that a single course of administration may not be enough to eliminate autoantibody-producing cells for platelets and ensure freedom from relapse (incidence of relapse; 43\%). A previous study also showed that Dex monotherapy was related to a higher incidence of posttherapeutic relapse (50\%), although the initial remission was high (85\%). ${ }^{15}$ Late relapse is one of the critical problems in ITP treatment, ${ }^{1-4}$ and there is an ongoing need to develop strategies to suppress relapse. Our findings indicated that additional courses of Dex (two to six total courses) and/or tapering PSL could significantly suppress relapse (21\%) compared with PSL (37\%).

There is no evidence to determine whether this superiority in Dex is due to the higher dosage of cumulative corticosteroid (two or more courses of Dex is equivalent to that of PSL administration for 1 month) or to specific pharmaceutical function of high-dose Dex which is lacking in PSL. It should be noted that the subgroup of patients treated with Dex including posttherapy were, as a consequence, administered the larger amounts of corticosteroid (1,000 $\mathrm{mg}$ or more as PSL) if compared with those treated with PSL arm irrespective of the estimated patient weight ( - Table 1 and - Table S5 [supplementary table available in the online version only]), indicating that corticosteroid dose itself may possibly be related to the outcome. On the other hand, superior SR in the Dex arm at 1 year in addition to 6 months may possibly imply the existence of specific effect in Dex, though not definitive. Further extended follow-up data will be helpful to conclude this point whether the Dex regimen induce real cures of ITP or simply delay the relapse due to its higher corticosteroid dosage. ${ }^{18}$

The lower incidence of adverse events in the Dex arm is also an important finding in the clinical setting. Adverse events due to corticosteroids are of concern because steroid-derived side effects can be obstacles in clinical courses of ITP therapy, especially in elderly people; ${ }^{10}$ the peaks of etiology in ITP exist in elderly people as well as in children. ${ }^{19}$ The incidence of steroid-derived adverse events, in general, depends greatly on both the average dose and cumulative duration of use, 20 indicating that it is essential to both complete whole courses of therapy in as a short period as possible and reduce the total amount of administered corticosteroids. In this respect, although the Dex arm required higher amounts of corticosteroids especially in the Dex with posttherapy subgroup, the lower frequency of adverse events was likely due to the shorter therapeutic periods. These analyses indicate that Dex therapy, even with the posttherapy including additional courses of Dex and/or tapering corticosteroid, may be tolerable as the initial therapy for newly diagnosed acute ITP. However, the grading of each adverse event could not be analyzed in the current study (due to a lack of enough information), and several RCTs ${ }^{7,8}$ did not sufficiently report the incidence of adverse events. Sensitivity analyses excluding these studies were not performed because of the small number of RCTs included. Moreover, subgroup analyses based on patient age were not performed for adverse events due to the lack of data; neuropsychiatric impact of high-dose Dex in the elderly should be paid enough attention in the future studies. ${ }^{21}$

Limitations of our study include the following: (1) data on long-term follow-up were not enough (not including late relapse), (2) data on quality of life were not included, (3) many studies showed high risk of bias in blinding, and (4) RCTs included in this analysis did not cover all the geographic regions of the world. The last limitation can cause heterogeneity in the analysis of long-term outcomes because accessibility to more novel and expensive agents (such as thrombopoietin receptor agonists) in case of nonremission to corticosteroid is totally different according to each region. The random-effects model used in our analysis may relieve such heterogeneity, but these limitations should be overcome with the future RCTs.

In conclusion, this systematic review and meta-analysis demonstrated the efficacy of Dex with posttherapy in newly diagnosed acute ITP; the results suggest that use of Dex instead of PSL may be more beneficial for patients with ITP. We have shown that Dex monotherapy is not enough to overwhelm the efficacy of PSL. The results were compatible between the fixedeffect model and the random-effects model. The corticosteroid regimens (including Dex) were not perfectly effective to all the patients; therefore, new strategies for treating ITP from the other mechanism than immune suppression, such as the use of thrombopoietin receptor agonists, should be evaluated in comparison with Dex, and future studies comparing Dex with these novel regimens are also essential to determine suitable initial therapeutic strategies for ITP.

Conflict of Interest

None declared.

\section{Contributions of Authors}

Conception and design: Y. Arai, H. Matsui, T. Jo, T. Kondo, A. Takaori-Kondo.

Analysis and interpretation of the data: Y. Arai, H. Matsui, T. Jo, T. Kondo.

Drafting of the article: Y. Arai.

Final approval of the article: Y. Arai, H. Matsui, T. Jo, T. Kondo, A. Takaori-Kondo.

\section{References}

1 Stasi R, Evangelista ML, Stipa E, Buccisano F, Venditti A, Amadori S. Idiopathic thrombocytopenic purpura: current concepts in pathophysiology and management. Thromb Haemost 2008; 99(01):4-13 
2 Cohen YC, Djulbegovic B, Shamai-Lubovitz O, Mozes B. The bleeding risk and natural history of idiopathic thrombocytopenic purpura in patients with persistent low platelet counts. Arch Intern Med 2000;160(11):1630-1638

3 Psaila B, Bussel JB. Immune thrombocytopenic purpura. Hematol Oncol Clin North Am 2007;21(04):743-759

4 Provan D, Stasi R, Newland AC, et al. International consensus report on the investigation and management of primary immune thrombocytopenia. Blood 2010;115(02):168-186

5 Neunert C, Lim W, Crowther M, Cohen A, Solberg L Jr, Crowther MA; American Society of Hematology. The American Society of Hematology 2011 evidence-based practice guideline for immune thrombocytopenia. Blood 2011;117(16):4190-4207

6 Cui ZG, Wei Y, Hou M, Zhao HG, Wang HY. [The efficacy and safety of 2 cycles' high-dose dexamethasone treatment adult primary immune thrombocytopenia]. Zhonghua Nei Ke Za Zhi 2011; 50(05):401-403

7 Mashhadi MA, Kaykhaei MA, Sepehri Z, Miri-Moghaddam E. Single course of high dose dexamethasone is more effective than conventional prednisolone therapy in the treatment of primary newly diagnosed immune thrombocytopenia. Daru 2012;20(01):7

8 Li ZY, Li DP, Yan ZL, et al. [Effect of different therapeutic regimens on regulatory $T$ cells in patients of primary immune thrombocytopenia]. Zhonghua Xue Ye Xue Za Zhi 2013;34(06):478-481

9 Bae SH, Ryoo H-M, Lee WS, et al. High dose dexamethasone vs. conventional dose prednisolone for adults with immune thrombocytopenia: a prospective multicenter phase III trial. Blood 2010; 116(21):Abstract 3687

10 Wei Y, Ji XB, Wang YW, et al. High-dose dexamethasone vs prednisone for treatment of adult immune thrombocytopenia: a prospective multicenter randomized trial. Blood 2016;127(03): 296-302

11 Mithoowani S, Gregory-Miller K, Goy J, et al. High-dose dexamethasone compared with prednisone for previously untreated primary immune thrombocytopenia: a systematic review and meta-analysis. Lancet Haematol 2016;3(10):e489-e496
12 Praituan W, Rojnuckarin P. Faster platelet recovery by high-dose dexamethasone compared with standard-dose prednisolone in adult immune thrombocytopenia: a prospective randomized trial. J Thromb Haemost 2009;7(06):1036-1038

13 Din B, Wang X, Shi Y, Li Y. Long-term effect of high-dose dexamethasone with or without low-dose dexamethasone maintenance in untreated immune thrombocytopenia. Acta Haematol 2015;133(01):124-128

14 Matschke J, Müller-Beissenhirtz H, Novotny J, et al. A randomized trial of daily prednisone versus pulsed dexamethasone in treatment-naïve adult patients with immune thrombocytopenia: EIS 2002 Study. Acta Haematol 2016;136(02):101-107

15 Cheng Y, Wong RS, Soo YO, et al. Initial treatment of immune thrombocytopenic purpura with high-dose dexamethasone. N Engl J Med 2003;349(09):831-836

16 Alpdogan O, Budak-Alpdogan T, Ratip S, et al. Efficacy of high-dose methylprednisolone as a first-line therapy in adult patients with idiopathic thrombocytopenic purpura. Br J Haematol 1998;103 (04):1061-1063

17 Liu XG, Ma SH, Sun JZ, et al. High-dose dexamethasone shifts the balance of stimulatory and inhibitory Fcgamma receptors on monocytes in patients with primary immune thrombocytopenia. Blood 2011;117(06):2061-2069

18 Cuker A, Prak ET, Cines DB. Can immune thrombocytopenia be cured with medical therapy? Semin Thromb Hemost 2015; 41(04):395-404

19 Moulis G, Palmaro A, Montastruc JL, Godeau B, Lapeyre-Mestre M, Sailler L. Epidemiology of incident immune thrombocytopenia: a nationwide population-based study in France. Blood 2014; 124(22):3308-3315

20 Huscher D, Thiele K, Gromnica-Ihle E, et al. Dose-related patterns of glucocorticoid-induced side effects. Ann Rheum Dis 2009; 68(07):1119-1124

21 Fang Q, Qian X, An J, Wen H, Cope DK, Williams JP. Higher dose dexamethasone increases early postoperative cognitive dysfunction. J Neurosurg Anesthesiol 2014;26(03):220-225 